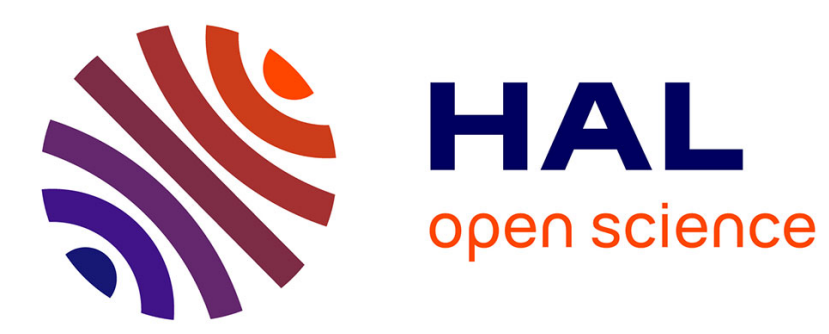

\title{
Global analysis of multi-strains SIS, SIR and MSIR epidemic models
}

\author{
Derdei Bichara, Abderrahman Iggidr, Gauthier Sallet
}

\section{To cite this version:}

Derdei Bichara, Abderrahman Iggidr, Gauthier Sallet. Global analysis of multi-strains SIS, SIR and MSIR epidemic models. Journal of Applied Mathematics and Computing, 2014, 44 (1-2), pp.273-292. 10.1007/s12190-013-0693-x . hal-00833057

\section{HAL Id: hal-00833057 https://hal.inria.fr/hal-00833057}

Submitted on 11 Jun 2013

HAL is a multi-disciplinary open access archive for the deposit and dissemination of scientific research documents, whether they are published or not. The documents may come from teaching and research institutions in France or abroad, or from public or private research centers.
L'archive ouverte pluridisciplinaire HAL, est destinée au dépôt et à la diffusion de documents scientifiques de niveau recherche, publiés ou non, émanant des établissements d'enseignement et de recherche français ou étrangers, des laboratoires publics ou privés. 


\title{
Global analysis of multi-strains SIS, SIR and MSIR epidemic models
}

\author{
D. Bichara A. Iggidr G. Sallet \\ EPI MASAIE \\ INRIA-Nancy Grand Est \& Université de Lorraine, Metz \\ LMAM(UMR CNRS 7122) \\ I.S.G.M.P. Bât A, \\ Ile du Saulcy, 57045 Metz Cedex 01, France. \\ \{derdei.bichara,abderrahman.iggidr,gauthier.sallet\}@inria.fr
}

\begin{abstract}
We consider SIS, SIR and MSIR models with standard mass action and varying population, with $n$ different pathogen strains of an infectious disease. We also consider the same models with vertical transmission. We prove that under generic conditions a competitive exclusion principle holds. To each strain a basic reproduction ratio can be associated. It corresponds to the case where only this strain exists. The basic reproduction ratio of the complete system is the maximum of each individual basic reproduction ratio.

Actually we also define an equivalent threshold for each strain. The winner of the competition is the strain with the maximum threshold. It turns out that this strain is the most virulent, i.e., this is the strain for which the endemic equilibrium gives the minimum population for the susceptible host population. This can be interpreted as a pessimization principle.
\end{abstract}

Keywords: Nonlinear dynamical systems, Global Stability, Lyapunov methods, Competition, Boundary equilibria

Mathematics Subject Classification (2000): 34A34, 34D23, 34D40, 92D30

\section{Introduction}

One of the most famous principles in theoretical ecology is the competitive exclusive principle, sometimes called Gause's Law of competitive exclusion, that stipulates: two species competing for the same resources cannot coexist indefinitely on the same ecological niche[15, 25, 28, 31]. However, according to Amstrong and McGehee [3], Volterra [38] was the first to use mathematical model to suggest that the indefinite coexistence of two or more species limited by the same resource is impossible. An abundance of literature was dedicated to the validation of this principle to epidemiological and/or host-parasite models $[1,2,3,4,6,7,8,15,18,19,24,25,28,29,30,31]$ and the references therein.

In mathematical epidemiology the seminal paper [6] proves a competitive exclusion of infection with different levels in single host populations. When only one strain is present a basic reproduction ratio can be computed $[13,36]$. Hence to each strain is associated such a number. The 
authors show that the competitive exclusion principle holds generically and that the winning strain is the one which maximizes its basic reproduction number. More precisely it is proved that all but one strain disappear, the winning strain being persistent. The model [6] considered by Bremermann and Thieme is a SIS model with $n$ strains.

Ackleh and Allen [1] consider an SIR model with $n$ strains and vertical transmission. They generalize a two-pathogen study by Andreasen and Pugliese [5]. They assume for the population dynamics a constant birth rate and a nonlinear death rate. Globally the population dynamics, without disease, is a generalized logistic type. They also assume a vertical transmission. The analysis is done for mass action incidence. The authors in [2] consider the SIS and SIR models with standard incidence. In both cases they derive sufficient conditions for competitive exclusion between the $n$ strains. One of these conditions involved the maximization of the basic reproduction ratio for the winning strain. As in the paper of Bremermann and Thieme this is a persistence result.

In [20], an analogous system has been considered with a general recruitment function $f(N)$, but without vertical transmission. Similar competitive exclusion results have been obtained (Theorem 3.3) under some additional assumptions. The stability of the endemic equilibria is obtained but unfortunately, as stated by the authors, the assumed hypotheses are generally hard to check. The study of the reduced system is still under investigation.

Recently attention has focused on understanding the factors that lead to coexistence or to competitive exclusion $[10,11,34]$. In the absence of multiple infections and the presence of complete cross-immunity, only the parasite strain persists that has the maximal basic reproduction ratio. See the survey [34]. This result has been proved under quite a few restrictions. However the validity of such a principle has been challenged. For example when the infection is transmitted both vertically and horizontally, simulations have shown that strains with lower virulence can out-compete strains with higher basic reproduction ratio [27].

To continue to explore this issue we consider a SIS or a SIR model with vertical and horizontal transmission and a different population dynamics. In our SIR model we, more generally, assume that when an individual recovers then only a fraction is cross-immunized. The other part of the individuals are again susceptible after recovering. We also show that MSIR model, where we consider protection by maternal antibodies, have the same properties as SIS and SIR models. We consider the bilinear mass action incidence. We assume, when acquired, total cross immunity and no superinfection. We prove that under generic conditions the principle of competitive exclusion applies. Compared to the other results this is not the basic reproduction ratio which determines the fate of the strain. We associate to each $i$ strain a threshold $\mathcal{T}_{0, i}$. This a threshold since $\mathcal{T}_{0, i}>1$ is equivalent to $\mathcal{R}_{0, i}>1$. The strain which maximizes its threshold wins the competition. Hence we can speak of a maximization of a threshold but not maximization of the basic reproduction ratio. We prove that an endemic equilibrium exists and that this equilibrium is globally asymptotically stable. Actually the winner is the strain which minimizes the number of susceptible at the equilibrium, which is not necessarily the strain which maximizes its basic reproduction ratio. A similar kind of phenomenon is observed in [19]. This can be seen as a pessimization principle $[12,33]$.

Most authors $[1,2,6]$ prove the competitive exclusion by persistence : under some conditions, all densities of infected strains, but one tend to 0 . The remaining strain stays positive, i.e., is persistent.

We consider in this paper, models with a different population dynamics. We consider SIS, SIR and MSIR models with horizontal and vertical transmission. We consider a constant recruitment (or immigration of susceptible individuals) and constant death rate, which gives the dynamics without disease $\dot{N}=\Lambda-\mu N$. 
Our law is less general but we can obtain more precise results. We always have a unique disease free equilibrium (DFE) and when the basic reproduction ratio is greater than one then some boundary endemic equilibria exist. Under a generic condition we prove that there is an equilibrium in a face of the nonnegative orthant, corresponding to the extinction of all the strains but one, which is globally asymptotically stable in the interior of the orthant and the corresponding face. We also describe the stability on each face. The evolution in a face of the orthant corresponds biologically to the nonexistence of some strains.

In this paper we prove this result by use of Lyapunov functions. Actually, the function we use is a "Volterra" like Lyapunov function. This kind of function has now been used successfully to ascertain global stability results in epidemiological models. See for example the references in $[14,35]$.

The paper is organized as follows :

In the next section we introduce the special SIS model, with vertical transmission. We compute the equilibria and the basic reproduction ratios. We also define an equivalent threshold.

Section 3 is devoted to the study of stability, under non-stringent generic hypothesis. We obtain global stability results on the biological domain. The last section examines the case of multi-strains SIR and MSIR model with vertical transmission.

\section{SIS model with vertical transmission}

\subsection{The model}

We assume that there is $n$ strains of pathogens. The host population is divided into susceptible and infected individuals. The number of susceptible individuals will be denoted by $S$, and the number of individuals infected with strain $i$ will be denoted by $I_{i}$. There is a mass action horizontal transmission. We assume that a fraction of the newborn, coming from the infectious individual, is infectious. In other words there is a vertical transmission. The infectious host ( by the $i$-th strain) gives birth to a new infected host at the rate $p_{i}$. Hence, $p_{i} I_{i}$ individuals enters into infected class $I_{i}$ and the same quantity is lacking from recruitment in the susceptible compartment. When recovered individuals are again susceptible to the disease.

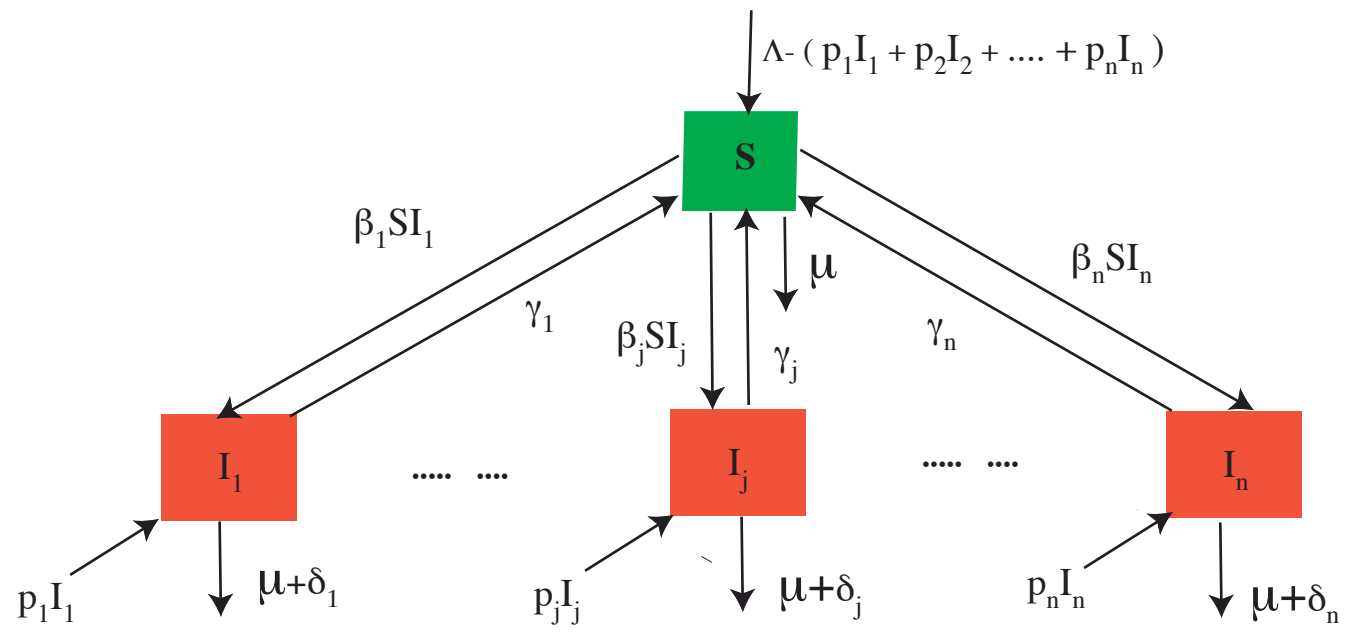

Figure 1: A SIS model with $n$ strains

The model is represented in Figure 1 and can be described by the following system of differential 
equations:

$$
\left\{\begin{array}{l}
\dot{S}=\Lambda-\sum_{i=1}^{n} \beta_{i} S I_{i}+\sum_{i=1}^{n}\left(\gamma_{i}-p_{i}\right) I_{i}-\mu S \\
\dot{I}_{i}=\beta_{i} S I_{i}-\left(\mu+\delta_{i}+\gamma_{i}-p_{i}\right) I_{i}, \text { for } i=1, \ldots, n .
\end{array}\right.
$$

The different parameters of our model are definite as:

$\Lambda$ : Recruitment of the susceptible individuals (birth, ...);

$\beta_{i}:$ transmission coefficient by $i$-th strain;

$\mu$ : Natural mortality rate;

$\delta_{i} \quad: \quad$ additional mortality of $i$-th strain;

$\gamma_{i}:$ recovered rate of $i$-th strain;

$p_{i}$ : rate at which an infectious host ( by the $i$-th strain) gives birth to a new infected host.

We assume that

$$
\mathbf{H}: \quad \gamma_{i} \geq p_{i} \text { for } i=1, \cdots, n
$$

This assumption ensures that the recruitment $\Lambda+\sum_{i=1}^{n}\left(\gamma_{i}-p_{i}\right) I_{i}$ in the susceptible compartment is always positive.

\section{$2.2 \quad$ Equilibria}

We denote by $N=S+\sum_{i=1}^{n} I_{i}$ the total host population. The evolution of $N$ is given by

$$
\dot{N}=\Lambda-\mu N-\sum_{i=1}^{n} \delta_{i} I_{i} \leq \Lambda-\mu N
$$

The region defined by

$$
\Omega=\left\{(S, I) \in \mathbb{R}_{+}^{n+1} \mid S+\sum_{i=1}^{n} I_{i} \leq \frac{\Lambda}{\mu}\right\}
$$

is a compact attracting positively invariant set for system (1).

The disease free equilibrium (DFE) is given by $\left(S^{*}, 0, \ldots, 0\right)$ with $S^{*}=\frac{\Lambda}{\mu}$. This equilibrium belongs to $\Omega$.

When only the strain $i$ exists, the model is a two dimensional system (with $S$ and $I_{i}$ ). The basic reproduction ratio $[13,36]$ of this model, with vertical transmission, is given by

$$
\mathcal{R}_{0, i}=\frac{\beta_{i} \frac{\Lambda}{\mu}+p_{i}}{\left(\mu+\delta_{i}+\gamma_{i}\right)}
$$

The system (1) has $n$ endemic equilibria, located in the boundary of the nonnegative orthant. Namely $\left(\bar{S}_{1}, \bar{I}_{1}, 0, \ldots, 0\right),\left(\bar{S}_{2}, 0, \bar{I}_{2}, 0, \ldots, 0\right), \ldots,\left(\bar{S}_{n}, 0, \ldots, 0, \bar{I}_{n}\right)$, where

$$
\bar{S}_{i}=\frac{\mu+\gamma_{i}+\delta_{i}-p_{i}}{\beta_{i}}, \quad \text { and } \quad \bar{I}_{i}=\frac{\Lambda}{\mu+\delta_{i}}\left(1-\frac{\mu\left(\mu+\gamma_{i}+\delta_{i}-p_{i}\right)}{\beta_{i} \Lambda}\right) .
$$

The boundary equilibrium $\left(\bar{S}_{i}, 0, \ldots, \bar{I}_{i}, \ldots, 0\right)$ is in $\Omega$ if and only if

$$
\mathcal{T}_{0, i}=\frac{\beta_{i} \Lambda}{\mu\left(\mu+\delta_{i}+\gamma_{i}-p_{i}\right)}>1
$$


It is clear, with our running hypothesis (2), that

$$
\mathcal{T}_{0, i}>1 \Longleftrightarrow \mathcal{R}_{0, i}>1
$$

Hence $\mathcal{T}_{0, i}$ is a threshold, in the sense of [21], and we have

$$
\bar{S}_{i}=\frac{S^{*}}{\mathcal{T}_{0, i}} \text {, and } \bar{I}_{i}=\frac{\Lambda}{\mu+\delta_{i}}\left(1-\frac{1}{\mathcal{T}_{0, i}}\right) \text {. }
$$

When $\mathcal{T}_{0, i}=1$ then $\bar{S}_{i}=S^{*}$ and $\bar{I}_{i}=0$.

A condition for a coexistence of strains $i$ and $j$ will be

$$
\frac{\mu+\gamma_{i}+\delta_{i}-p_{i}}{\beta_{i}}=\frac{\mu+\gamma_{j}+\delta_{j}-p_{j}}{\beta_{j}} .
$$

or equivalently

$$
\mathcal{T}_{0, i}=\mathcal{T}_{0, j}>1
$$

To have a coexistence of all the strains in the interior of the nonnegative orthant will require $n$ preceding equalities. This condition is clearly non generic. In this case, there is a continuum of stable equilibria. The proof is developed at the subsection 3.3.

The coexistence can occur if the death rate is density dependent $[1,5]$ or even with a standard incidence law and exponentially growing host populations [26].

\section{Global stability analysis}

Let us recall that we assume, without loss of generality, our standing hypothesis (2). We define

$$
\mathcal{R}_{0}=\max _{i=1, \ldots, n} \mathcal{R}_{0, i}, \quad \text { and } \mathcal{T}_{0}=\max _{i=1, \cdots, n} \mathcal{T}_{0, i}
$$

We have seen that

$$
\mathcal{T}_{0}>1 \Longleftrightarrow \mathcal{R}_{0}>1
$$

\subsection{Global stability of the DFE}

\section{Theorem 3.1}

If $\mathcal{T}_{0} \leq 1$, the DFE is globally asymptotically stable in the nonnegative orthant. If $\mathcal{T}_{0}>1$, the DFE is unstable.

\section{Proof.}

We consider the Lyapunov function

$$
V=\sum_{i=1}^{n} I_{i}
$$

We will use the LaSalle invariance principle on compact sets $[16,22,23]$ to prove the asymptotic stability. By hypothesis each $\mathcal{T}_{0, i}$ satisfies $\mathcal{T}_{0, i} \leq 1$. 
Computing the derivatives of $V$ along the trajectories gives

$$
\begin{aligned}
\dot{V} & =\sum_{i=1}^{n}\left(\beta_{i} S-\left(\mu+\delta_{i}+\gamma_{i}-p_{i}\right)\right) I_{i}, \\
& \leq \sum_{i=1}^{n}\left(\beta_{i} \frac{\Lambda}{\mu}-\left(\mu+\delta_{i}+\gamma_{1}-p_{i}\right)\right) I_{i}, \\
& \leq \sum_{i=1}^{n}\left(\mu+\delta_{i}+\gamma_{i}-p_{i}\right)\left(\mathcal{T}_{0, i}-1\right) I_{i}, \\
& \leq 0 .
\end{aligned}
$$

Now we consider the set contained in $\Omega$ where $\dot{V}=0$.

To have $\dot{V}=0$ in $\Omega$ this implies that for each index

$$
I_{i}=0 \text { or } S=\bar{S}_{i}
$$

We associate to each subset $\mathcal{I}$ of indices, a point defined by if $j \notin \mathcal{I}$ then $I_{j}=0$ and if $i \in \mathcal{I}$ then $S=\bar{S}_{i}$ and for any couple $(i, k)$ in $\mathcal{I}^{2}$ we assume $\bar{S}_{i}=\bar{S}_{k}$. For each kind of subset and condition we have a solution of $\dot{V}=0$. All these solutions constitute a set $\mathcal{E}$. We state, from the relation (3), that our condition implies $\mathcal{T}_{0, i}=\mathcal{T}_{0, k}$. Then we only consider the subset of indices $\mathcal{I}$ such that for any couple $(j, k) \in \mathcal{I}^{2}, \mathcal{T}_{0, j}=\mathcal{T}_{0, k}$. Any subset of this kind gives a solution in $\mathcal{E}$.

But now we consider the greatest invariant set contained in $\Omega$ and in $\mathcal{E}$.

A trajectory starting from one of this point is given by

$$
\dot{S}=\Lambda-\bar{S}_{i} \sum_{i \in \mathcal{I}} \beta_{i} I_{i}+\sum_{i \in \mathcal{I}}\left(\gamma_{i}-p_{i}\right) I_{i}-\mu \bar{S}_{i}
$$

We state that for any solution in $\mathcal{E}$ we have $\dot{I}_{i}=0$.

By invariance $\dot{S}=0$, hence

$$
\Lambda-\mu \frac{S^{*}}{\mathcal{T}_{0, i}}=\sum_{i \in \mathcal{I}}\left(\beta_{i} \frac{S^{*}}{\mathcal{T}_{0, i}}-\left(\gamma_{i}-p_{i}\right)\right) I_{i}=\sum_{i \in \mathcal{I}}\left(\mu+\delta_{i}\right) I_{i}
$$

Now we recall that $\mathcal{T}_{0} \leq 1$, hence each $\mathcal{T}_{0, i} \leq 1$, which implies $\Lambda-\mu \frac{S^{*}}{\mathcal{T}_{0, i}} \leq 0$.

If $\mathcal{T}_{0, i}<1$, the preceding inequality cannot be satisfied in the nonnegative orthant. Finally, we see that our set of indices $\mathcal{I}$ is such that $\mathcal{T}_{0, i}=1$ for any index in $\mathcal{I}$. But this implies again $I_{i}=0$ by invariance. Then the only invariant set contained in $\Omega$, such that $\dot{V}=0$, is the DFE. This proves, by the results of $[22,23]$, that the DFE is globally asymptotically stable in $\Omega$. Since $\Omega$ is an attracting set the stability is global in the nonnegative orthant. 
Now, suppose that $\mathcal{T}_{0}>1$ (or equivantly $\mathcal{R}_{0}>1$ ). The Jacobian matrix of system (1) at the DFE is:

$$
J_{0}=\left(\begin{array}{ccccc}
-\mu & -\beta_{1} S^{*}+\left(\gamma_{1}-p_{1}\right) & -\beta_{2} S^{*}+\left(\gamma_{2}-p_{2}\right) & \cdots & -\beta_{n} S^{*}+\left(\gamma_{n}-p_{n}\right) \\
0 & \beta_{1} S^{*}-\alpha_{1} & 0 & \cdots & 0 \\
\vdots & \ddots & \ddots & \ddots & \vdots \\
0 & \ddots & \ddots & \beta_{i} S^{*}-\alpha_{i} & \vdots \\
\vdots & \cdots & \ddots & \ddots & 0 \\
0 & \cdots & \cdots & 0 & \beta_{n} S^{*}-\alpha_{n}
\end{array}\right)
$$

with $\alpha_{i}=\mu+\delta_{i}+\gamma_{i}-p_{i}$. The eigenvalues of $J_{0}$ are $-\mu$ and $\alpha_{i}\left(\mathcal{T}_{0, i}-1\right)$. Hence if $\mathcal{T}_{0}>1$, then the Jacobian matrix $J_{0}$ has at least one positive eigenvalue and therefore the DFE is unstable.

\section{Remark 3.1}

We have proved the result without any additional hypotheses on the relations between the $\mathcal{T}_{0, i}$.

\subsection{Global stability and competitive exclusion}

In this section we assume $\mathcal{R}_{0}>1$ or equivalently $\mathcal{T}_{0}>1$. We will assume in the sequel that one strain is maximizing its threshold. In other words there is a strain (we can assume that it is the one with index 1) such that for any $i>1$ we have

$$
\mathcal{T}_{0,1}>\mathcal{T}_{0, i} .
$$

With $\mathcal{T}_{0}>1$, let $i_{0}$ the last index for which $\mathcal{T}_{0, i}>1$. Then

$$
\mathcal{T}_{0,1}>\mathcal{T}_{0,2} \geq \cdots \mathcal{T}_{0, i_{0}}>1 \geq \mathcal{T}_{0, i_{0}+1} \geq \cdots \mathcal{T}_{0, n}
$$

\section{Theorem 3.2}

Under the hypothesis $\mathcal{T}_{0,1}>\mathcal{T}_{0, i}$ satisfied for $i=2, \cdots, n$, the endemic equilibrium

$$
\left(\frac{S^{*}}{\mathcal{T}_{0,1}}, \frac{\Lambda}{\mu+\delta_{1}}\left(1-\frac{1}{\mathcal{T}_{0,1}}\right), 0, \cdots, 0\right),
$$

is globally asymptotically stable on the intersection of the of the nonnegative orthant with the two half-hyperspace defined by the inequalities $S>0$ and $I_{1}>0$.

\section{Proof:}

We denote by $(\bar{S}, \bar{I})$, with $\bar{I}=\left(\frac{\Lambda}{\mu+\delta_{1}}\left(1-\frac{1}{\mathcal{T}_{0,1}}\right), 0, \cdots, 0\right) \in \mathbb{R}_{+}^{n}$ the endemic equilibrium.

We consider the following Lyapunov function, defined on the intersection of the nonnegative orthant with the half open hyperplane spaces given by the inequalities $S>0$ and $I_{1}>0$ :

$$
\begin{aligned}
V(S, I)=S-\bar{S} \log S+\frac{\mu+\delta_{1}}{\mu+\delta_{1}+\gamma_{1}-p_{1}}\left(I_{1}-\bar{I}_{1} \log I_{1}\right) & \\
& +\sum_{i=2}^{n}\left(1-\frac{\gamma_{i}-p_{i}}{\beta_{i} \bar{S}}\left(\frac{\mathcal{T}_{0, i}}{\mathcal{T}_{0,1}}\right)^{2}\right) I_{i}+K .
\end{aligned}
$$


Where $K$ is chosen such that $V(\bar{S}, \bar{I})=0$.

$$
K=-\bar{S}+\bar{S} \log \bar{S}-\frac{\mu+\delta_{1}}{\mu+\delta_{1}+\gamma_{1}-p_{1}}\left(\bar{I}_{1}-\bar{I}_{1} \log \bar{I}_{1}\right) .
$$

Indeed this function, on the considered domain, is a positive definite Lyapunov function. To sustain that claim, we must prove that the coefficients of $I_{i}$ are positive. For this issue we use the following relation

$$
\frac{\mathcal{T}_{0, i}}{\mathcal{T}_{0,1}}=\frac{\frac{\beta_{i} \Lambda}{\mu\left(\mu+\delta_{i}+\gamma_{i}-p_{i}\right)}}{\frac{\beta_{1} \Lambda}{\mu\left(\mu+\delta_{1}+\gamma_{1}-p_{1}\right)}}=\frac{\beta_{i} \bar{S}}{\mu+\delta_{i}+\gamma_{i}-p_{i}} .
$$

From this relation, we deduce the inequality

$$
\frac{\gamma_{i}-p_{i}}{\beta_{i} \bar{S}}\left(\frac{\mathcal{T}_{0, i}}{\mathcal{T}_{0,1}}\right)^{2}=\frac{\gamma_{i}-p_{i}}{\mu+\delta_{i}+\gamma_{i}-p_{i}} \frac{\mathcal{T}_{0, i}}{\mathcal{T}_{0,1}}<1
$$

Using the endemic relation $\Lambda=\beta_{1} \bar{S} \bar{I}_{1}-\left(\gamma_{1}-p_{1}\right) \bar{I}_{1}+\mu \bar{S}$, the derivative of $\mathrm{V}$ along trajectories of system (1) is

$$
\begin{aligned}
\dot{V}=\left(1-\frac{\bar{S}}{S}\right) \dot{S}+\frac{\mu+\delta_{1}}{\mu+\delta_{1}+\gamma_{1}-p_{1}}\left(1-\frac{\bar{I}_{1}}{I_{1}}\right) \dot{I}_{1} & \\
& +\underbrace{\sum_{i=2}^{n}\left(1-\frac{\gamma_{i}-p_{i}}{\beta_{i} \bar{S}}\left(\frac{\mathcal{T}_{0, i}}{\mathcal{T}_{0,1}}\right)^{2}\right)\left(\beta_{i} S I_{i}-\left(\mu+\delta_{i}+\gamma_{i}-p_{i}\right) I_{i}\right)}_{C} .
\end{aligned}
$$

Which can also be written

$$
\begin{aligned}
& \dot{V}= \beta_{1} \bar{S} \bar{I}_{1}-\left(\gamma_{1}-p_{1}\right) \bar{I}_{1}+\mu \bar{S}-\beta_{1} S I_{1}-\sum_{i=2}^{n} \beta_{i} S I_{i}+\left(\gamma_{1}-p_{1}\right) I_{1} \\
&+\sum_{i=2}^{n}\left(\gamma_{i}-p_{i}\right) I_{i}-\mu S \\
&-\frac{\bar{S}}{S}\left(\beta_{1} \bar{S} \bar{I}_{1}-\left(\gamma_{1}-p_{1}\right) \bar{I}_{1}+\mu \bar{S}-\beta_{1} S I_{1}-\sum_{i=2}^{n} \beta_{i} S I_{i}+\left(\gamma_{1}-p_{1}\right) I_{1}\right. \\
&\left.+\sum_{i=2}^{n}\left(\gamma_{i}-p_{i}\right) I_{i}-\mu S\right) \\
&+\frac{\mu+\delta_{1}}{\mu+\delta_{1}+\gamma_{1}-p_{1}}\left[\beta_{1} S I_{1}-\left(\mu+\delta_{1}+\gamma_{1}-p_{1}\right) I_{1}-\beta_{1} S \bar{I}_{1}\right. \\
&\left.+\left(\mu+\delta_{1}+\gamma_{1}-p_{1}\right) \bar{I}_{1}\right]+C \\
&= {\left[\mu \bar{S}+\left(\mu+\delta_{1}\right) \bar{I}_{1}+\left(\gamma_{1}-p_{1}\right) I_{1}\right]\left(2-\frac{S}{\bar{S}}-\frac{\bar{S}}{S}\right)+} \\
& \sum_{i=2}^{n}\left(\gamma_{i}-p_{i}-\beta_{i} S-\frac{\bar{S}}{S}\left(\gamma_{i}-p_{i}-\beta_{i} S\right)\right) I_{i}+C
\end{aligned}
$$


Finally we decompose $\dot{V}$ in the sum of three expressions :

$$
\begin{aligned}
\dot{V}=\left[\mu \bar{S}+\left(\mu+\delta_{1}\right) \bar{I}_{1}+\left(\gamma_{1}-p_{1}\right) I_{1}\right]\left(2-\frac{S}{\bar{S}}-\frac{\bar{S}}{S}\right)+ & \\
& \sum_{i=2}^{n}\left(\gamma_{i}-p_{i}-\beta_{i} S-\frac{\bar{S}}{S}\left(\gamma_{i}-p_{i}-\beta_{i} S\right)\right) I_{i}+C .
\end{aligned}
$$

By using the relation

$$
\frac{\mathcal{T}_{0, i}}{\mathcal{T}_{0,1}}=\frac{\frac{\beta_{i} \Lambda}{\mu\left(\mu+\delta_{i}+\gamma_{i}-p_{i}\right)}}{\frac{\beta_{1} \Lambda}{\mu\left(\mu+\delta_{1}+\gamma_{1}-p_{1}\right)}}=\frac{\beta_{i} \bar{S}}{\mu+\delta_{i}+\gamma_{i}-p_{i}}
$$

We have:

$$
C=\sum_{i=2}^{n}\left(1-\frac{\gamma_{i}-p_{i}}{\beta_{i} \bar{S}}\left(\frac{\mathcal{T}_{0, i}}{\mathcal{T}_{0,1}}\right)^{2}\right)\left[\beta_{i} S I_{i}-\left(\mu+\delta_{i}+\gamma_{i}-p_{i}\right) I_{i}\right]
$$

Which can be written

$$
C=\sum_{i=2}^{n}\left(\beta_{i} S-\beta_{i} \bar{S}\left(\frac{\mathcal{T}_{0,1}}{\mathcal{T}_{0, i}}\right)-\left(\gamma_{i}-p_{i}\right) \frac{S}{\bar{S}}\left(\frac{\mathcal{T}_{0, i}}{\mathcal{T}_{0,1}}\right)^{2}+\left(\gamma_{i}-p_{i}\right)\left(\frac{\mathcal{T}_{0, i}}{\mathcal{T}_{0,1}}\right)\right) I_{i}
$$

We call $B$ the sum of the last two terms in $\dot{V}$, i.e.,

$$
B=\sum_{i=2}^{n}\left(\gamma_{i}-p_{i}-\beta_{i} S-\frac{\bar{S}}{S}\left(\gamma_{i}-p_{i}-\beta_{i} S\right)\right) I_{i}+C
$$

Then, using relation (6)

$$
\begin{aligned}
B & =\sum_{i=2}^{n}\left[\gamma_{i}-p_{i}-\beta_{i} S-\frac{\bar{S}}{S}\left(\gamma_{i}-p_{i}\right)+\beta_{i} \bar{S}\right] I_{i} \\
& +\sum_{i=2}^{n}\left[\beta_{i} S-\beta_{i} \bar{S}\left(\frac{\mathcal{T}_{0,1}}{\mathcal{T}_{0, i}}\right)-\left(\gamma_{i}-p_{i}\right) \frac{S}{\bar{S}}\left(\frac{\mathcal{T}_{0, i}}{\mathcal{T}_{0,1}}\right)^{2}+\left(\gamma_{i}-p_{i}\right)\left(\frac{\mathcal{T}_{0, i}}{\mathcal{T}_{0,1}}\right)\right] I_{i}
\end{aligned}
$$

Thus,

$$
\begin{array}{r}
B=\sum_{i=2}^{n}\left[\left(\gamma_{i}-p_{i}\right)+\beta_{i} \bar{S}-\frac{\bar{S}}{S}\left(\gamma_{i}-p_{i}\right)-\beta_{i} \bar{S}\left(\frac{\mathcal{T}_{0,1}}{\mathcal{T}_{0, i}}\right)-\left(\gamma_{i}-p_{i}\right) \frac{S}{\bar{S}}\left(\frac{\mathcal{T}_{0, i}}{\mathcal{T}_{0,1}}\right)^{2}\right. \\
\left.+\left(\gamma_{i}-p_{i}\right)\left(\frac{\mathcal{T}_{0, i}}{\mathcal{T}_{0,1}}\right)\right] I_{i}
\end{array}
$$

Using the inequality between the geometric and arithmetic means we have

$$
-\frac{\bar{S}}{S}\left(\gamma_{i}-p_{i}\right)-\left(\gamma_{i}-p_{i}\right) \frac{S}{\bar{S}}\left(\frac{\mathcal{T}_{0, i}}{\mathcal{T}_{0,1}}\right)^{2} \leq-2 \sqrt{\left(\gamma_{i}-p_{i}\right)^{2}\left(\frac{\mathcal{T}_{0, i}}{\mathcal{T}_{0,1}}\right)^{2}}
$$


Hence, with the hypothesis $\gamma_{i} \geq p_{i}$, from the preceding inequality we deduce

$$
\begin{gathered}
B \leq \sum_{i=2}^{n}\left[-2\left(\gamma_{i}-p_{i}\right) \frac{\mathcal{T}_{0, i}}{\mathcal{T}_{0,1}}+\gamma_{i}-p_{i}+\beta_{i} \bar{S}-\beta_{i} \bar{S} \frac{\mathcal{T}_{0,1}}{\mathcal{T}_{0, i}}+\left(\gamma_{i}-p_{i}\right) \frac{\mathcal{T}_{0, i}}{\mathcal{T}_{0,1}}\right] I_{i} \\
\leq \sum_{i=2}^{n}\left[\left(\gamma_{i}-p_{i}\right)\left(1-\frac{\mathcal{T}_{0, i}}{\mathcal{T}_{0,1}}\right)+\beta_{i} \bar{S}\left(1-\frac{\mathcal{T}_{0,1}}{\mathcal{T}_{0, i}}\right)\right] I_{i} .
\end{gathered}
$$

Since $\gamma_{i}-p_{i} \leq \mu+\gamma_{i}+\delta_{i}-p_{i}=\beta_{i} \bar{S} \frac{\mathcal{T}_{0,1}}{\mathcal{T}_{0, i}}$, with $\frac{\mathcal{T}_{0,1}}{\mathcal{T}_{0, i}}>1$, we can write, for each index $i=2, \cdots, n$, the following inequalities

$$
\begin{aligned}
{\left[\left(\gamma_{i}-p_{i}\right)\left(1-\frac{\mathcal{T}_{0, i}}{\mathcal{T}_{0,1}}\right)+\beta_{i} \bar{S}\left(1-\frac{\mathcal{T}_{0,1}}{\mathcal{T}_{0, i}}\right)\right] } & I_{i} \leq \\
& \left(\beta_{i} \bar{S} \frac{\mathcal{T}_{0,1}}{\mathcal{T}_{0, i}}\left(1-\frac{\mathcal{T}_{0, i}}{\mathcal{T}_{0,1}}\right)+\beta_{i} \bar{S}\left(1-\frac{\mathcal{T}_{0,1}}{\mathcal{T}_{0, i}}\right)\right) I_{i}=0 .
\end{aligned}
$$

This relation proves that $B \leq 0$. Then $\dot{V}$ is bounded by the first expression

$$
\dot{V} \leq\left[\mu \bar{S}+\left(\mu+\delta_{1}\right) \bar{I}_{1}+\left(\gamma_{1}-p_{1}\right) I_{1}\right]\left(2-\frac{S}{\bar{S}}-\frac{\bar{S}}{S}\right) .
$$

Since $\gamma_{1} \geq p_{1}$, using again the inequality between the arithmetic and geometric means, we obtain $\dot{V} \leq 0$.

By Lyapunov's theorem this proves the stability of the endemic equilibrium.

To prove the asymptotic stability, we will use LaSalle's principle [16, 22, 23]. We recall the expression of $\dot{V}$

$$
\begin{aligned}
\dot{V}=\left(\mu \bar{S}+\left(\mu+\delta_{1}\right) \bar{I}_{1}+\left(\gamma_{1}-p_{1}\right) I_{1}\right) & \left(2-\frac{S}{\bar{S}}-\frac{\bar{S}}{S}\right) \\
& +\sum_{i=2}^{n}\left(\gamma_{i}-p_{i}-\beta_{i} S-\frac{\bar{S}}{S}\left(\gamma_{i}-p_{i}-\beta_{i} S\right)\right) I_{i}+C .
\end{aligned}
$$

We have to find the points $\left(S, I_{1}, \cdots, I_{n}\right)$ for which $\dot{V}=0$.

We have seen that $V$ is the sum of three nonpositive quantities. The first term, a positive definite function of $S$, is zero if and only if $S=\bar{S}$.

The second term, with $S=\bar{S}$, is equal to zero since

$$
\left.\sum_{i=2}^{n}\left(\gamma_{i}-p_{i}-\beta_{i} S-\frac{\bar{S}}{S}\left(\gamma_{i}-p_{i}-\beta_{i} S\right)\right) I_{i}\right|_{S=\bar{S}}=0
$$


Finally,

$$
\begin{aligned}
C & =\sum_{i=2}^{n}\left(1-\frac{\gamma_{i}-p_{i}}{\beta_{i} \bar{S}}\left(\frac{\mathcal{T}_{0, i}}{\mathcal{T}_{0,1}}\right)^{2}\right)\left(\beta_{i} S I_{i}-\left(\mu+\delta_{i}+\gamma_{i}-p_{i}\right) I_{i}\right) \\
& =\sum_{i=2}^{n}\left(1-\frac{\gamma_{i}-p_{i}}{\beta_{i} \bar{S}}\left(\frac{\mathcal{T}_{0, i}}{\mathcal{T}_{0,1}}\right)^{2}\right)\left(\beta_{i} \bar{S}-\left(\mu+\delta_{i}+\gamma_{i}-p_{i}\right)\right) I_{i} \\
& =\sum_{i=2}^{n}\left(1-\frac{\gamma_{i}-p_{i}}{\beta_{i} \bar{S}}\left(\frac{\mathcal{T}_{0, i}}{\mathcal{T}_{0,1}}\right)^{2}\right)\left(\beta_{i} \bar{S}-\beta_{i} \bar{S} \frac{\mathcal{T}_{0,1}}{\mathcal{T}_{0, i}}\right) I_{i} \\
& =\sum_{i=2}^{n} \underbrace{\left(1-\frac{\gamma_{i}-p_{i}}{\beta_{i} \bar{S}}\left(\frac{\mathcal{T}_{0, i}}{\mathcal{T}_{0,1}}\right)^{2}\right)}_{>0} \beta_{i} \bar{S} \underbrace{\left(1-\frac{\mathcal{T}_{0,1}}{\mathcal{T}_{0, i}}\right)}_{<0} I_{i} .
\end{aligned}
$$

Then $C=0$ if and only each $I_{i}=0$ for $i=2, \cdots, n$.

We found that $\dot{V}=0$ implies $S=\bar{S}$ and $I_{i}=0$ for $i=2, \cdots, n$. We conclude by Lasalle's principle, that the greatest invariant set is reduced to the endemic equilibrium. This ends the proof of the Theorem .

For any strain, we have defined $\mathcal{T}_{0, i}=\frac{\beta_{i} \Lambda}{\mu\left(\mu+\gamma_{i}+\delta_{i}-p_{i}\right)}=\frac{\Lambda}{\mu \bar{S}_{i}}$.

We showed, that the winner strain maximizes the threshold $\mathcal{T}_{0, i}$, thus minimizes $\bar{S}_{i}$. This result is analogous to those obtained in [19]. This can be interpreted like a pessimization principle $[12]$.

\subsection{The coexistence case}

The coexistence of all the strains occurs when the following non generic condition holds:

$$
\forall(i, j) \in\{1, \ldots, n\}^{2}, \quad \mathcal{T}_{0, i}=\mathcal{T}_{0, j}>1
$$

When the $n$ equalities are satisfied, we have:

$$
\begin{aligned}
\bar{S} & =\frac{\mu+\gamma_{i}+\delta_{i}-p_{i}}{\beta_{i}} \quad \forall i \in\{1, \ldots, n\} \\
& =\frac{\Lambda}{\mu \mathcal{T}_{0,1}}
\end{aligned}
$$

and then $\bar{I}_{1}, \ldots, \bar{I}_{n}$ satisfy the linear algebraic equation (using the equation of $\dot{S}$ ):

$$
\Lambda-\sum_{i=1}^{n} \beta_{i} \frac{\Lambda}{\mu \mathcal{T}_{0,1}} \bar{I}_{i}+\sum_{i=1}^{n}\left(\gamma_{i}-p_{i}\right) \bar{I}_{i}-\mu \frac{\Lambda}{\mu \mathcal{T}_{0,1}}=0
$$

Hence, we have a continuum equilibria of (1) given by the following set:

$$
\mathcal{S}=\left\{\left(\frac{\Lambda}{\mu \mathcal{T}_{0,1}}, \bar{I}_{1}, \ldots, \bar{I}_{n}\right) \in \Omega \mid \Lambda-\sum_{i=1}^{n} \beta_{i} \frac{\Lambda}{\mu \mathcal{T}_{0,1}} \bar{I}_{i}+\sum_{i=1}^{n}\left(\gamma_{i}-p_{i}\right) \bar{I}_{i}-\mu \frac{\Lambda}{\mu \mathcal{T}_{0,1}}=0\right\}
$$

Knowing that $\mathcal{T}_{0,1}=\mathcal{T}_{0, i}$, we have the following equivalence: 


$$
\left(\frac{\Lambda}{\mu \mathcal{T}_{0,1}}, \bar{I}_{1}, \ldots, \bar{I}_{n}\right) \in \mathcal{S} \Longleftrightarrow \sum_{i=1}^{n}\left(\mu+\delta_{i}\right) \bar{I}_{i}=\Lambda-\mu \frac{\Lambda}{\mu \mathcal{T}_{0,1}}
$$

\section{Theorem 3.3}

Suppose that the condition $\mathcal{T}_{0,1}=\mathcal{T}_{0, i}>1$ is satisfied for all $i=2, \cdots, n$. The equilibrium set $\mathcal{S}$ is globally asymptotically stable.

\section{Proof:}

Let us consider the following Lyapunov function:

$$
V\left(S, I_{1}, \ldots, I_{n}\right)=S-\bar{S}-\bar{S} \log \frac{S}{\bar{S}}+\sum_{i=1}^{n} \frac{\mu+\delta_{i}}{\mu+\delta_{i}+\gamma_{i}-p_{i}}\left(I_{i}-\bar{I}_{i}-\bar{I}_{i} \log \frac{I_{i}}{\bar{I}_{i}}\right) .
$$

First recall that the following relation holds:

$$
1=\frac{\mathcal{T}_{0, i}}{\mathcal{T}_{0,1}}=\frac{\frac{\beta_{i} \Lambda}{\mu\left(\mu+\delta_{i}+\gamma_{i}-p_{i}\right)}}{\frac{\beta_{1} \Lambda}{\mu\left(\mu+\delta_{1}+\gamma_{1}-p_{1}\right)}}=\frac{\beta_{i} \bar{S}}{\mu+\delta_{i}+\gamma_{i}-p_{i}} .
$$

The derivative of $\mathrm{V}$ along trajectories of system (1) is

$$
\begin{gathered}
\dot{V}=\left(1-\frac{\bar{S}}{S}\right) \dot{S}+\sum_{i=1}^{n} \frac{\mu+\delta_{i}}{\mu+\delta_{i}+\gamma_{i}-p_{i}}\left(1-\frac{\bar{I}_{i}}{I_{i}}\right) \dot{I}_{i} . \\
\dot{V}=\Lambda-\sum_{i=1}^{n} \beta_{i} S I_{i}+\sum_{i=1}^{n}\left(\gamma_{i}-p_{i}\right) I_{i}-\mu S-\frac{\bar{S}}{S}\left(\Lambda-\sum_{i=1}^{n} \beta_{i} S I_{i}+\sum_{i=1}^{n}\left(\gamma_{i}-p_{i}\right) I_{i}-\mu S\right) \\
+\sum_{i=1}^{n} \frac{\mu+\delta_{i}}{\mu+\delta_{i}+\gamma_{i}-p_{i}}\left[\beta_{i} S I_{i}-\left(\mu+\delta_{i}+\gamma_{i}-p_{i}\right) I_{i}-\beta_{i} S \bar{I}_{i}+\left(\mu+\delta_{i}+\gamma_{i}-p_{i}\right) \bar{I}_{i}\right] . \\
=\Lambda-\sum_{i=1}^{n} \beta_{i} S I_{i}+\sum_{i=1}^{n}\left(\gamma_{i}-p_{i}\right) I_{i}-\mu S-\Lambda \frac{\bar{S}}{S}+\sum_{i=1}^{n} \beta_{i} \bar{S} I_{i}-\sum_{i=1}^{n}\left(\gamma_{i}-p_{i}\right) I_{i} \frac{\bar{S}}{S}+\mu \bar{S} \\
+\sum_{i=1}^{n}\left[\frac{\mu+\delta_{i}}{\mu+\delta_{i}+\gamma_{i}-p_{i}} \beta_{i} S I_{i}-\left(\mu+\delta_{i}\right) I_{i}-\frac{\mu+\delta_{i}}{\mu+\delta_{i}+\gamma_{i}-p_{i}} \beta_{i} S \bar{I}_{i}+\left(\mu+\delta_{i}\right) \bar{I}_{i}\right] .
\end{gathered}
$$

Using $\beta_{i} \bar{S}=\mu+\delta_{i}+\gamma_{i}-p_{i}$, and $\Lambda=\sum_{i=1}^{n} \beta_{i} \bar{S} \bar{I}_{i}-\sum_{i=1}^{n}\left(\gamma_{i}-p_{i}\right) \bar{I}_{i}+\mu \bar{S}=\sum_{i=1}^{n}\left(\mu+\delta_{i}\right) \bar{I}_{i}+\mu \bar{S}$

$$
\begin{aligned}
\dot{V}= & \Lambda-\sum_{i=1}^{n} \beta_{i} S I_{i}+\sum_{i=1}^{n}\left(\gamma_{i}-p_{i}\right) I_{i}-\mu S-\Lambda \frac{\bar{S}}{S}+\sum_{i=1}^{n} \beta_{i} \bar{S} I_{i}-\sum_{i=1}^{n}\left(\gamma_{i}-p_{i}\right) I_{i} \frac{\bar{S}}{S}+\mu \bar{S} \\
& +\sum_{i=1}^{n}\left(\mu+\delta_{i}\right)\left[I_{i} \frac{S}{\bar{S}}-I_{i}-\bar{I}_{i} \frac{S}{\bar{S}}+\bar{I}_{i}\right] \cdot \\
= & 2 \Lambda-\Lambda \frac{\bar{S}}{S}-\frac{S}{\bar{S}}\left(\mu \bar{S}+\sum_{i=1}^{n}\left(\mu+\delta_{i}\right) \bar{I}_{i}\right)+\sum_{i=1}^{n}\left(\gamma_{i}-p_{i}-\left(\mu+\delta_{i}\right)+\beta_{i} \bar{S}\right) I_{i} \\
& -\sum_{i=1}^{n}\left(\gamma_{i}-p_{i}\right) I_{i} \frac{\bar{S}}{S}-\sum_{i=1}^{n} \beta_{i} \bar{S} \frac{S}{\bar{S}} I_{i}+\sum_{i=1}^{n}\left(\mu+\delta_{i}\right) I_{i} \frac{S}{\bar{S}} \\
= & \Lambda\left(2-\frac{\bar{S}}{S}-\frac{S}{\bar{S}}\right)+\sum_{i=1}^{n}\left(\gamma_{i}-p_{i}\right) I_{i}\left(2-\frac{\bar{S}}{S}-\frac{S}{\bar{S}}\right)
\end{aligned}
$$


Hence,

$$
\dot{V}=\underbrace{\left(\Lambda+\sum_{i=1}^{n}\left(\gamma_{i}-p_{i}\right) I_{i}\right)}_{D}\left(2-\frac{S}{\bar{S}}-\frac{\bar{S}}{S}\right)
$$

Thanks to Assumption 2, $D>0$.

Hence $\dot{V} \leq 0$ for all $\left(S, I_{1}, \ldots I_{n}\right) \in \Omega$, and $\dot{V}=0$ if and only if $S=\bar{S}$. By Lyapunov theorem, the equilibria $\left(\bar{S}, \bar{I}_{1}, \bar{I}_{2}, \cdots, \bar{I}_{n}\right)$ where $\bar{S}$ is fixed by (8) and $\bar{I}_{i}$ for $i=1, \ldots, n$, belong to $\mathcal{S}$, are stable. It is clear that the only invariant set contained in $\Omega$ such that $\dot{V}=0$ is reduced to $\mathcal{S}$. Hence, by LaSalle's invariance principle, the set of equilibria $\mathcal{S}$ is globally asymptotically stable. This ends the proof.

\section{SIR and MSIR models}

\subsection{SIR model}

We consider a SIR model where a proportion of infected can recover and have total immunity for all strains. For this model (see Fig. 2), we have the following system:

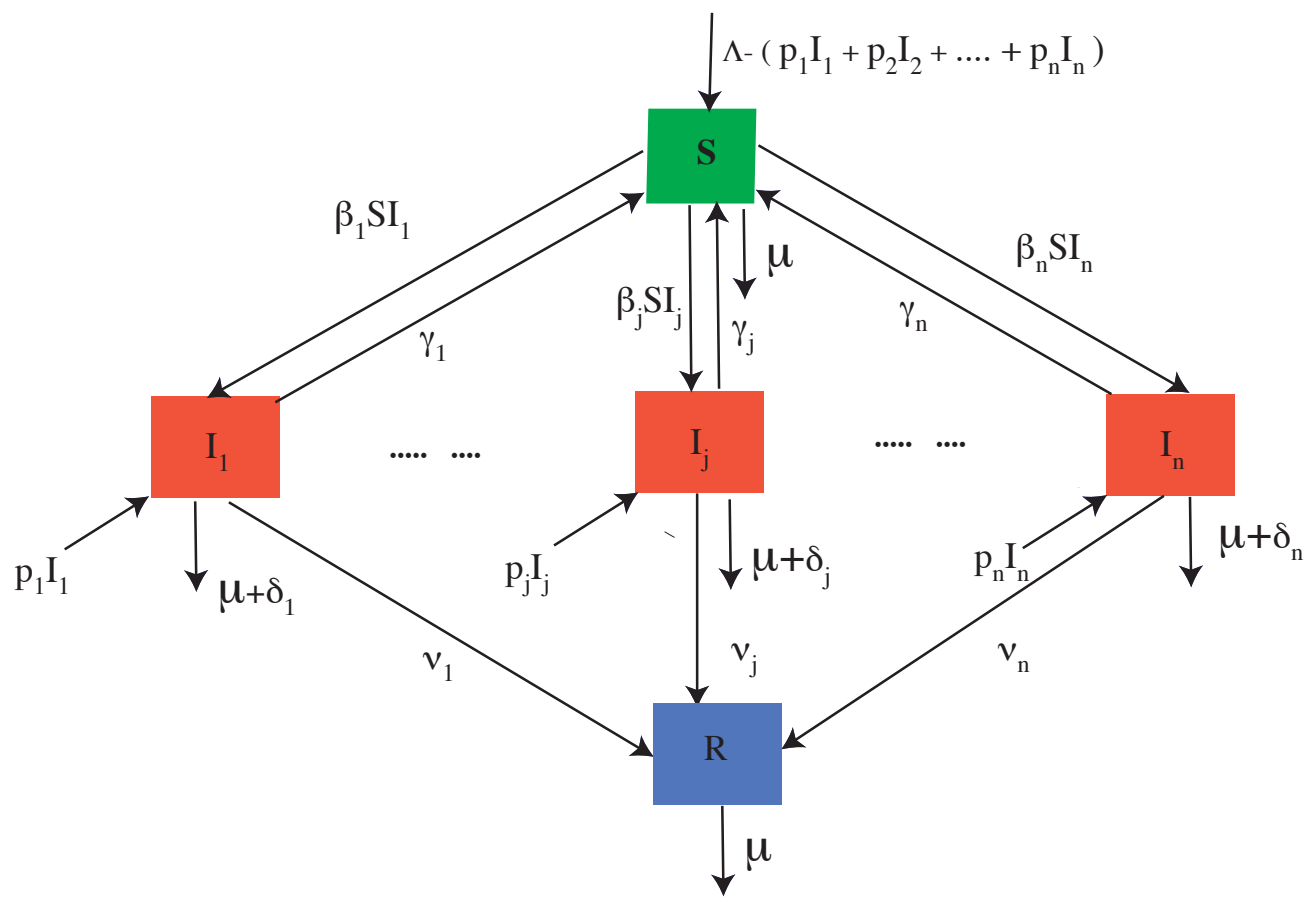

Figure 2: A SIR model with $n$ strains

$$
\left\{\begin{array}{l}
\dot{S}=\Lambda-\sum_{i=1}^{n} \beta_{i} S I_{i}+\sum_{i=1}^{n}\left(\gamma_{i}-p_{i}\right) I_{i}-\mu S \\
\dot{I}_{i}=\beta_{i} S I_{i}-\left(\mu+\delta_{i}+\nu_{i}+\gamma_{i}-p_{i}\right) I_{i} \quad i=1, \ldots, n \\
\dot{R}=\sum_{i=1}^{n} \nu_{i} I_{i}-\mu R
\end{array}\right.
$$


Since $R$ is not present in the $n+1$ first equations, we have a triangular system. We are in a compact domain. By Vidyasagar 's theorem [37], to study stability, it is sufficient to study the system composed by the $n+1$ first equations. Then the above results concerning multi-strains SIS system (1) are valid for the SIR system (12) with the same Lyapunov functions by replacing $\delta_{i}$ by $\delta_{i}^{\prime}=\delta_{i}+\nu_{i}$.

\subsection{MSIR model}

In practice, for some diseases, a proportion of newborns may have temporary passive immunity due to protection from maternal antibodies. Thus we need to incorporate an additional class $M$ which contains these infants with passive immunity [17]. If the maternal antibodies disappear from the body, the infant moves to the susceptible class $S[17,32]$. So, if we suppose, for $0 \leq q<1$, that the quantity $q \Lambda$ are the infants with passive immunity and $(1-q) \Lambda$ are the ones without passive immunity, we have the following graph:

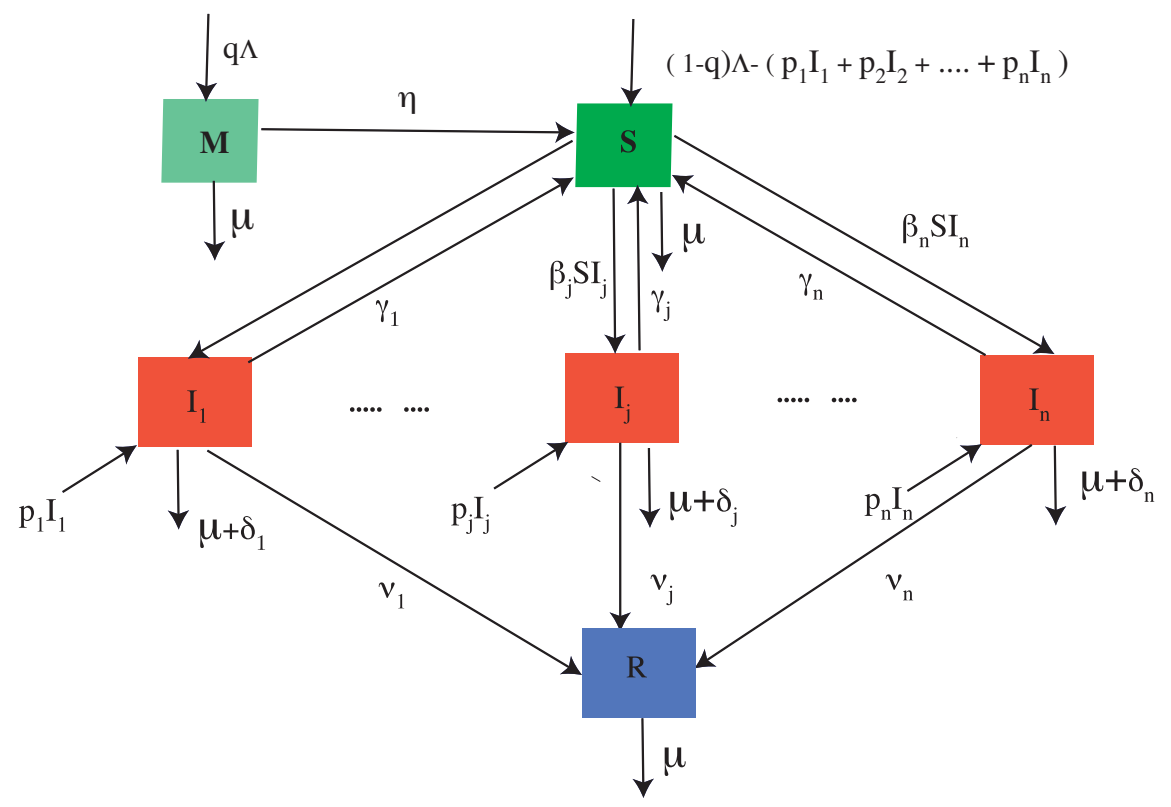

Figure 3: A MSIR model with $n$ strains

The dynamics of this model is given by:

$$
\left\{\begin{array}{l}
\dot{M}=q \Lambda-(\mu+\eta) M \\
\dot{S}=(1-q) \Lambda-\sum_{i=1}^{n} \beta_{i} S I_{i}+\sum_{i=1}^{n}\left(\gamma_{i}-p_{i}\right) I_{i}-\mu S+\eta M \\
\dot{I}_{i}=\beta_{i} S I_{i}-\left(\mu+\delta_{i}+\nu_{i}+\gamma_{i}-p_{i}\right) I_{i} \quad i=1, \ldots, n \\
\dot{R}=\sum_{i=1}^{n} \nu_{i} I_{i}-\mu R
\end{array}\right.
$$

This system is clearly triangular and the first equation of $M$ satisfies:

$$
\limsup _{t \rightarrow+\infty} M(t)=\frac{q \Lambda}{\mu+\eta}:=M^{*}
$$


Thanks to a result by Thieme and Castillo-Chavez [9], the system (13) have the same qualitative dynamics as the following limit system:

$$
\left\{\begin{array}{l}
\dot{S}=(1-q) \Lambda-\sum_{i=1}^{n} \beta_{i} S I_{i}+\sum_{i=1}^{n}\left(\gamma_{i}-p_{i}\right) I_{i}-\mu S+\eta M^{*} \\
\dot{I}_{i}=\beta_{i} S I_{i}-\left(\mu+\delta_{i}+\nu_{i}+\gamma_{i}-p_{i}\right) I_{i} \quad i=1, \ldots, n \\
\dot{R}=\sum_{i=1}^{n} \nu_{i} I_{i}-\mu R
\end{array}\right.
$$

This system can be rewritten as

$$
\left\{\begin{array}{l}
\dot{S}=\tilde{\Lambda}-\sum_{i=1}^{n} \beta_{i} S I_{i}+\sum_{i=1}^{n}\left(\gamma_{i}-p_{i}\right) I_{i}-\mu S \\
\dot{I}_{i}=\beta_{i} S I_{i}-\left(\mu+\delta_{i}+\nu_{i}+\gamma_{i}-p_{i}\right) I_{i} \quad i=1, \ldots, n \\
\dot{R}=\sum_{i=1}^{n} \nu_{i} I_{i}-\mu R
\end{array}\right.
$$

where $\tilde{\Lambda}=\frac{\Lambda}{\mu+\eta}(\eta+(1-q) \mu)$. This system (15) has the exact same properties as the system (12).

\section{Simulations}

In this section we show some figures obtained by numerical simulation of the previous results. Figure 4 presents the case when $\mathcal{T}_{0} \leq 1$, i.e., when the the DFE is GAS. The non generic case where coexistence occurs is represented in Figure 5 and Figure 6. The Figure 7 illustrates the generic case when the exclusion holds. 


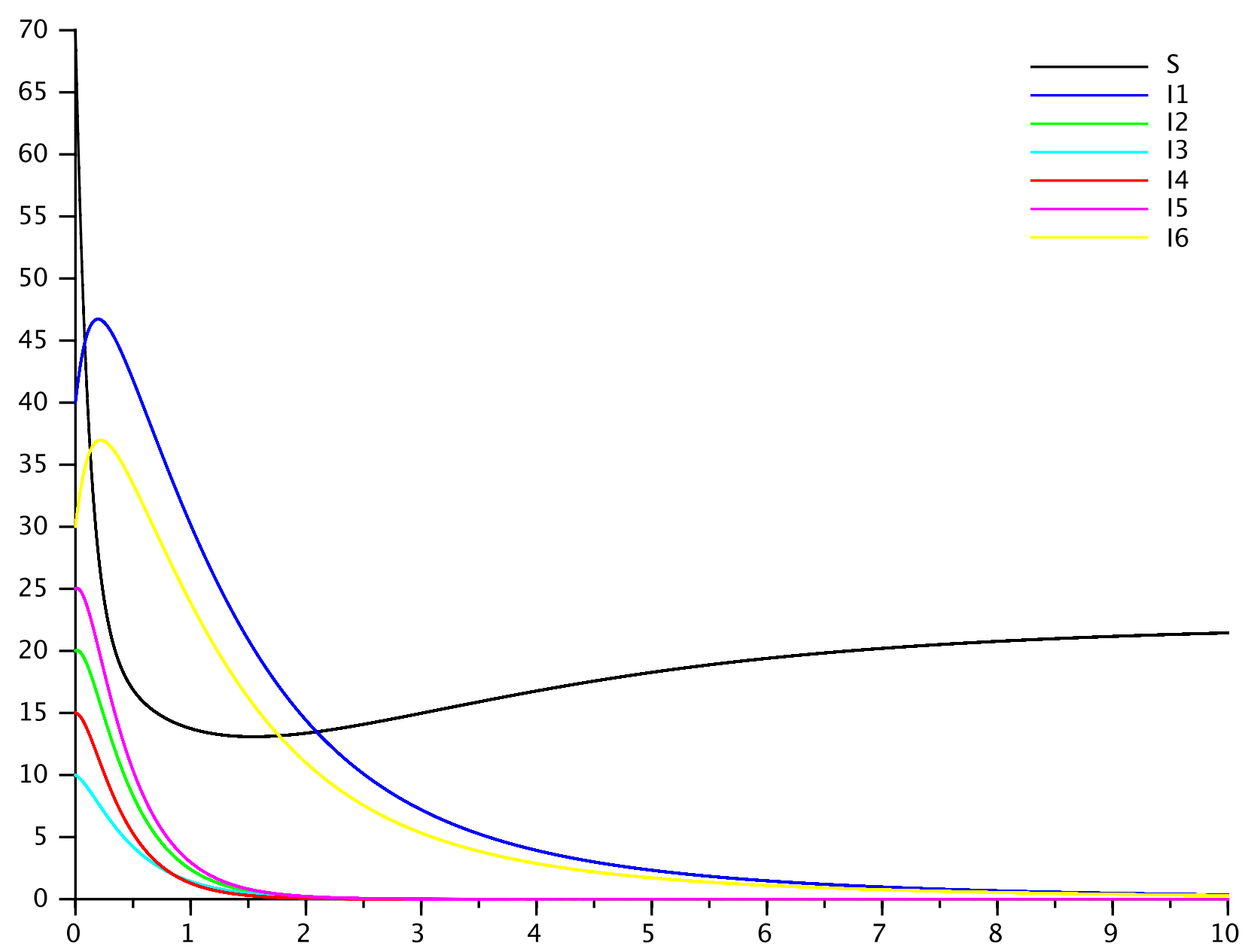

Figure 4: Case of DFE where all strains extinct. The values of different parameters are the following $\Lambda=20, \beta_{1}=0.05, \beta_{2}=0.05, \beta_{3}=0.03, \beta_{4}=0.05, \beta_{5}=0.05, \beta_{6}=0.06, \mu=$ $0.9 ; \gamma_{1}=0.6, \gamma_{3}=0.9, \gamma_{5}=1.4, \gamma_{2}=1.5, \gamma_{4}=1.9, \gamma_{6}=0.7, p_{1}=0.3, p_{2}=0.01, p_{3}=$ $0.03, p_{4}=0.025, p_{5}=0.03, p_{6}=0.08, \delta_{1}=0.2, \delta_{3}=0.85, \quad \delta 5=0.98, \delta_{2}=0.85, \delta_{4}=$ $0.8, \delta_{6}=0.05$. We have the inequality $\mathcal{T}_{i}<1 \forall i \in\{1, \ldots, 6\}$.

Here we present the non generic case where, with the same parameters and different initial conditions, the system (1) converges toward two different coexistence equilibria. However all of these equilibria belong to the set $\mathcal{S}$.

With parameters $\Lambda=15, \beta_{1}=0.98 \beta_{2}=0.98, \mu=0.1 ; \gamma_{1}=0.52, \gamma_{2}=0.72, p_{1}=0.2, p_{2}=$ $0.2, \delta_{1}=0.62, \delta_{2}=0.42$, we have the following equalities: $\mathcal{T}_{1}=\mathcal{T}_{2}=14.134615$. We have also $\bar{S} \approx 1.0612245$. 


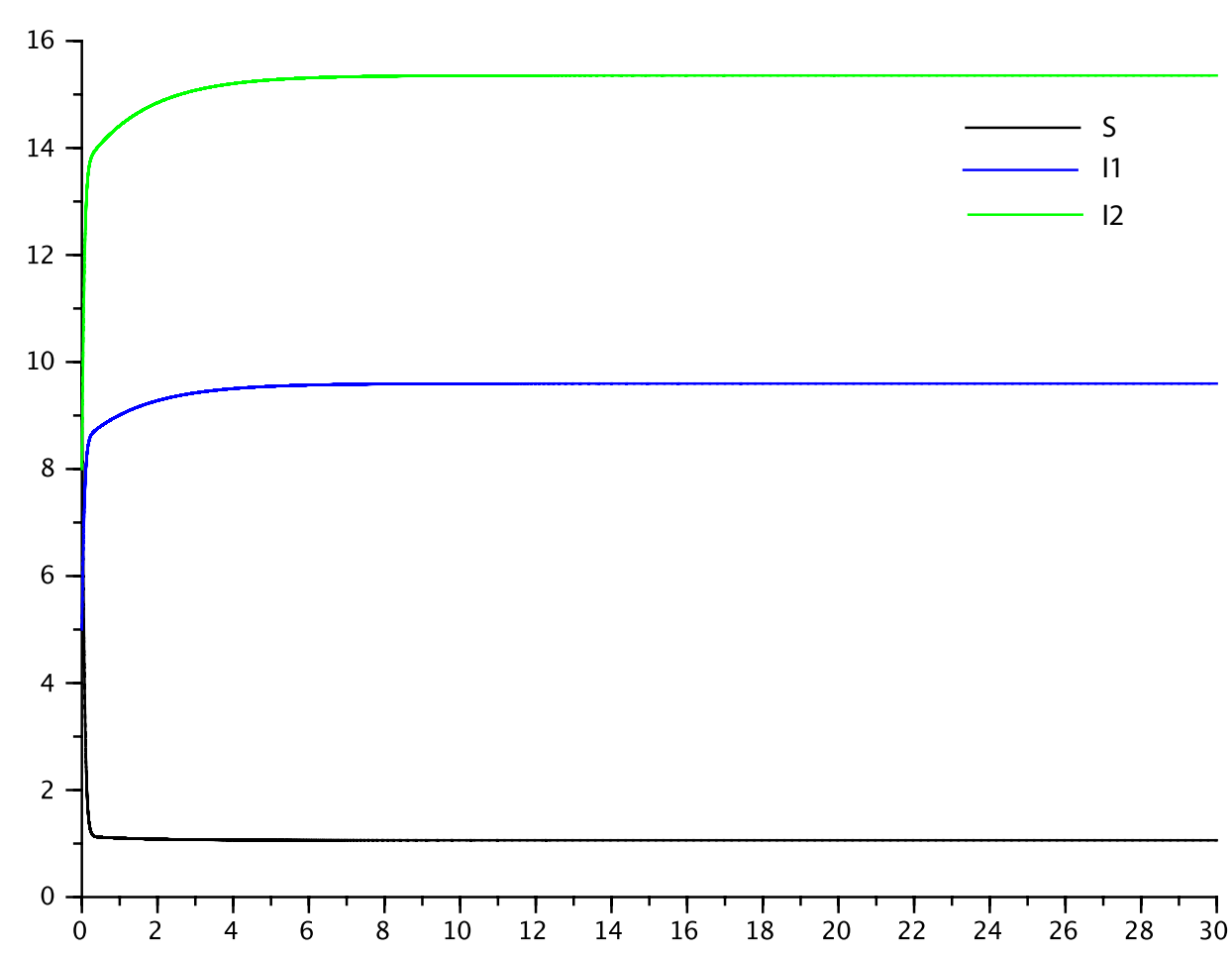

Figure 5: Non generic case: An SIS model with 2 coexisting strains. For the initial condition $\left(S(0), I_{1}(0), I_{2}(0)\right)=(10,5,8)$, this figure shows that the system (1) towards the equilibrium $\bar{S} \approx 1.0612245, \bar{I}_{1} \approx 9.5965706$ and $\bar{I}_{2} \approx 15.354513$. Obviously, the relation (9) holds: $\Lambda-(\mu+$ $\left.\delta_{1}\right) \bar{I}_{1}-\left(\mu+\delta_{2}\right) \bar{I}_{2}-\mu \bar{S} \approx 0$.

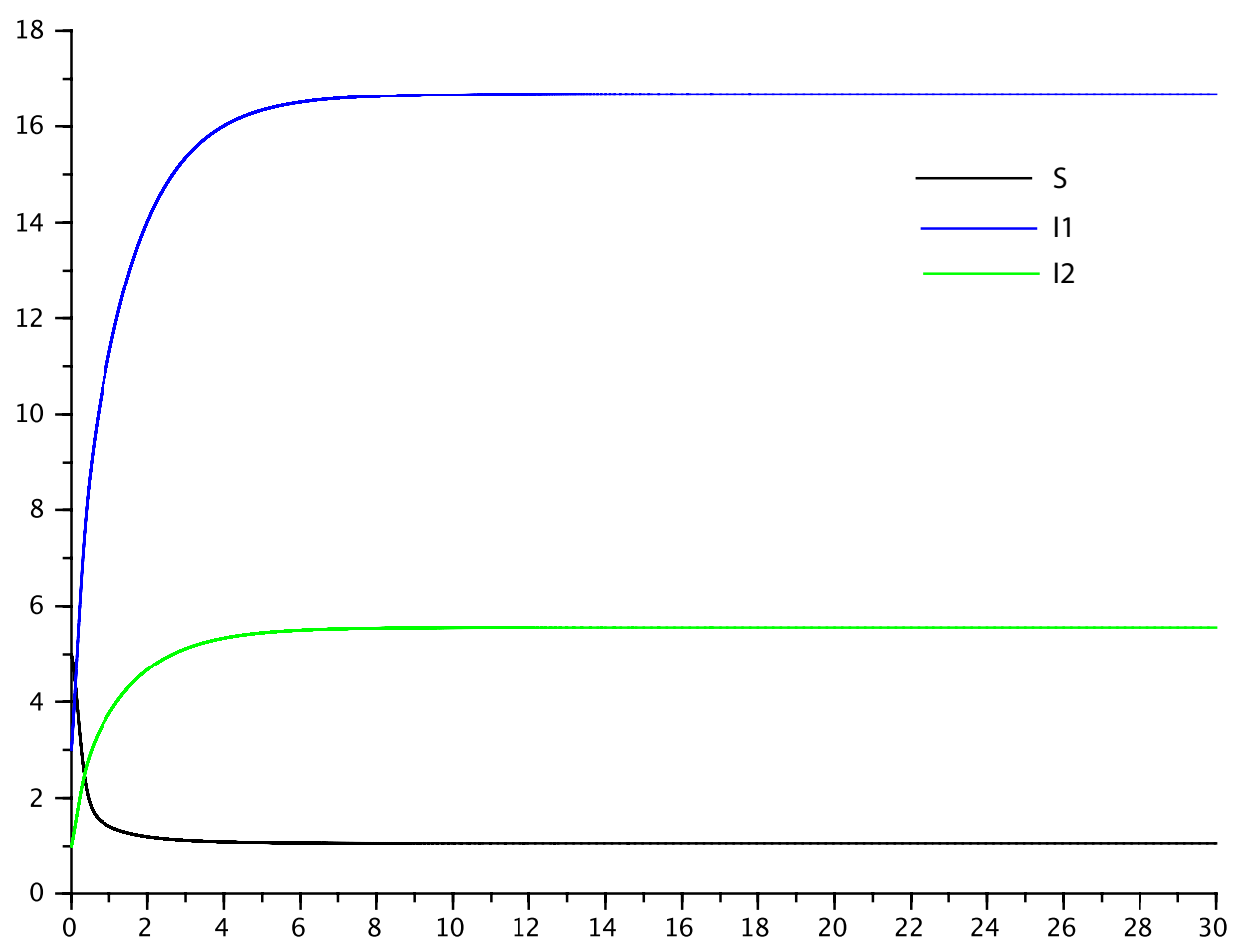

Figure 6: Non generic case: An SIS model with 2 coexisting strains. For the initial condition $\left(S(0), I_{1}(0), I_{2}(0)\right)=(5,3,1)$, this figure shows that the system (1) towards the equilibrium $\bar{S} \approx 1.0612245, \bar{I}_{1} \approx 16.672251$ and $\bar{I}_{2} \approx 5.5574169$. Again, even with completely different values of $\bar{I}_{1}$ and $\bar{I}_{2}$ from the Figure (5), the relation (9) holds: $\Lambda-\left(\mu+\delta_{1}\right) \bar{I}_{1}-\left(\mu+\delta_{2}\right) \bar{I}_{2}-\mu \bar{S} \approx 0$. 


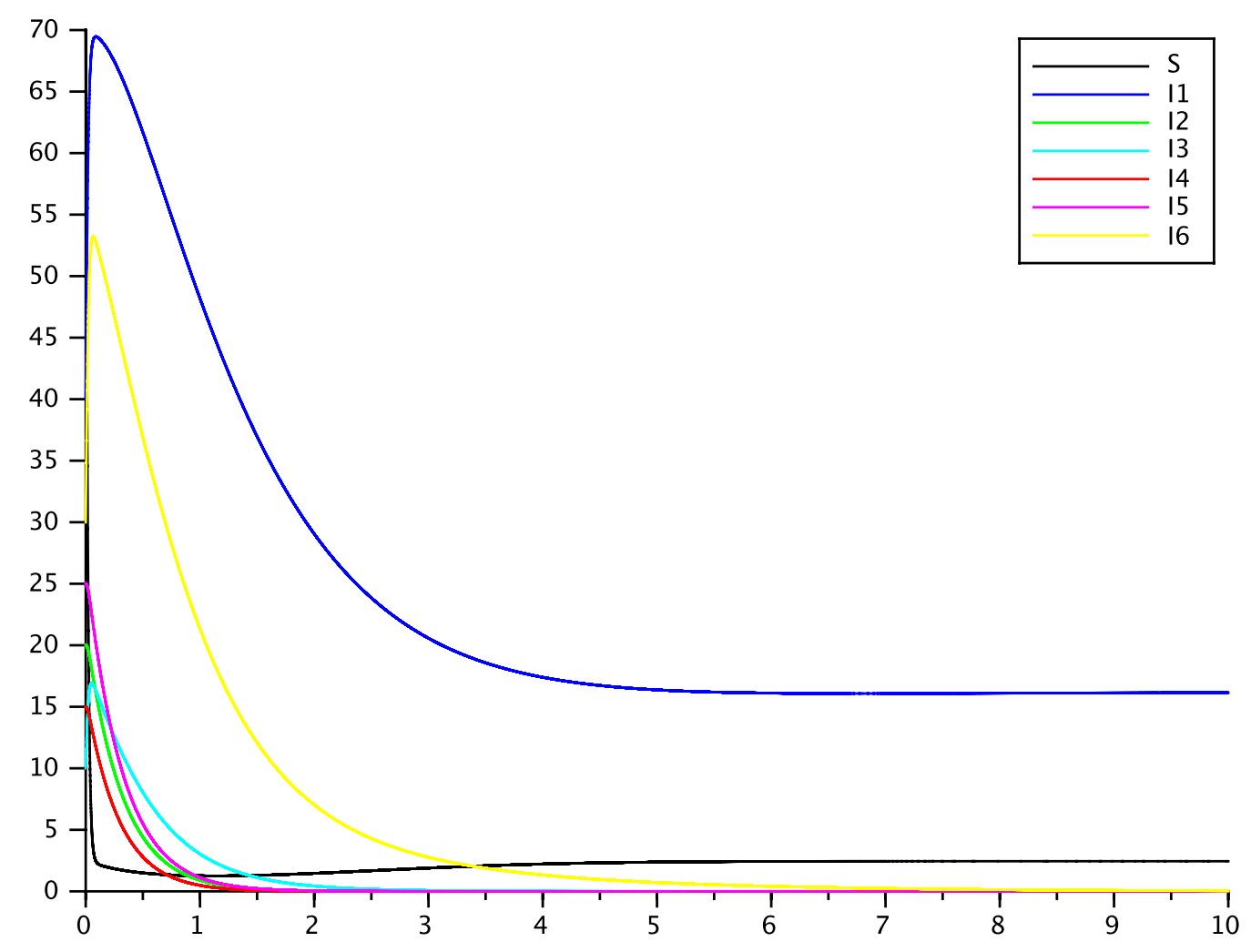

Figure 7: Generic case where an exclusive competition occurs. With parameters $\Lambda=20, \beta_{1}=$ $0.45, \beta_{2}=0.05, \beta_{3}=0.5, \beta_{4}=0.05, \beta_{5}=0.05, \beta_{6}=0.5, \mu=0.9 ; \gamma_{1}=0.3, \gamma_{3}=0.9, \gamma_{5}=$ $1.4, \gamma_{2}=1.5, \gamma_{4}=1.9, \gamma_{6}=0.9, p_{1}=0.3, p_{2}=0.01, p_{3}=0.03, p_{4}=0.025, p_{5}=0.03, p_{6}=$ $0.08, \delta_{1}=0.2, \delta_{3}=0.85, \delta_{5}=0.98, \delta_{2}=0.85, \delta_{4}=0.8, \delta_{6}=0.05$., we have the following inequalities: $\mathcal{T}_{1}>\mathcal{T}_{6}>\mathcal{T}_{3}>1>\mathcal{T}_{2}>\mathcal{T}_{4}>\mathcal{T}_{5}$. The strain \# 1 wins the competition while other strains disappear.

acknowledgements We would like to thank two anonymous referees for helpful comments and suggestions which led to an improvement of the presentation of this manuscript.

\section{References}

[1] A. S. Ackleh And L. J. S. Allen, Competitive exclusion and coexistence for pathogens in an epidemic model with variable population size, J. Math. Biol., 47, pp. 153-168 (2003).

[2] A. S. Ackleh And L. J. S. Allen, Competitive exclusion in sis and sir epidemic models with total cross immunity and density-dependent host mortality, Discrete Contin. Dyn. Syst. Ser. B, 5, pp. 175-188 (2005).

[3] R. A. Amstrong and R. McGehee, Competitive exclusion, The American Naturalist, 115, pp. 151-170 (1980).

[4] V. Andreasen, J. Lin, And S. A. Levin, The dynamics of cocirculating influenza strains conferring partial cross-immunity, J. Math. Biol., 35, pp. 825-842 (1997). 
[5] V. Andreasen And A. Pugliese, Pathogen coexistence induced by density-dependent host mortality, J. Theoret. Biol., pp. 159-165 (1995).

[6] H. Bremermann and H. R. Thieme, A competitive exclusion principle for pathogen virulence, J. Math. Biol., pp. 179-190 (1989).

[7] C. Castillo-Chavez, W. Huang, And J. Li, Competitive exclusion in gonorrhea models and other sexually transmitted diseases, SIAM J. Appl. Math., 56, pp. 494-508 (1996).

[8] _ Competitive exclusion and coexistence of multiple strains in an SIS STD model, SIAM J. Appl. Math., 59, pp. 1790-1811 (electronic) (1999).

[9] C. Castillo-Chavez and H. R. Thieme. Asymptotically autonomous epidemic models. In O. Arino, D. Axelrod, M. Kimmel, and M. Langlais, editors, Math. Pop. Dyn.: Analysis of heterogeneity, volume Vol. One: Theory of Epidemics, pages 33-50. Wuerz Pub, Winnipeg, Canada, 1995.

[10] T. Dhirasakdanon And H. R. Thieme, Persistence of vertically transmitted parasite strains which protect against more virulent horizontally transmitted strains, in Modeling and dynamics of infectious diseases, vol. 11 of Ser. Contemp. Appl. Math. CAM, Higher Ed. Press, Beijing, pp. 187-215 (2009).

[11] T. Dhirasakdanon and H. R. Thieme, Stability of the endemic coexistence equilibrium for one host and two parasites, Math. Model. Nat. Phenom., 5, pp. 109-138 (2010),.

[12] O. Diekmann, A beginner's guide to adaptive dynamics, in Mathematical modelling of population dynamics, vol. 63 of Banach Center Publ., Polish Acad. Sci., Warsaw, pp. 47-86 (2004).

[13] O. Diekmann, J. A. P. Heesterbeek, And J. A. J. Metz, On the definition and the computation of the basic reproduction ratio $R_{0}$ in models for infectious diseases in heterogeneous populations, J. Math. Biol., 28, pp. 365-382 (1990).

[14] A. Fall, A. Iggidr, G. Sallet, And J. J. Tewa, Epidemiological models and Lyapunov functions, Math. Model. Nat. Phenom., 2, pp. 55-73 (2007).

[15] G. Gause, The struggle for existence, Williams and Wilkins, reprinted 1964 Hafner, (1934).

[16] J. HaLE, Ordinary differential equations, Krieger, (1980).

[17] H. W. Heтнсоте, The mathematics of infectious diseases, SIAM Rev., 42 (2000), pp. 599-653 (electronic).

[18] S. Hsu, H. Smith, And P. Waltman, Competitive exclusion and coexistence for competitive systems on ordered banach spaces, Trans. Amer. Math. Soc., 348 (1996).

[19] A. Iggidr, J.-C. Kamgang, G. Sallet, And J.-J. Tewa, Global analysis of new malaria intrahost models with a competitive exclusion principle, SIAM J. Appl. Math., 67, pp. 260-278 (electronic) (2006).

[20] S. IwAmi And T. HARA, Global stability of a generalized epidemic model, J. Math. analysis applic., 362, pp. 286-300 (2010). 
[21] J. A. Jacquez, C. P. Simon, And J. Koopman, Core groups and the r0s for subgroups in heterogeneous sis and si models, in Epidemics models : their structure and relation to data, D. ed., ed., Cambridge University Press, pp. 279-301 (1996).

[22] J. LASALLE, Stability theory for ordinary differential equations. stability theory for ordinary differential equations., J. Differ. Equations, 41, pp. 57-65 (1968).

[23] J. P. LASAlle, The stability of dynamical systems, Society for Industrial and Applied Mathematics, Philadelphia, Pa., 1976. With an appendix: "Limiting equations and stability of nonautonomous ordinary differential equations" by Z. Artstein, Regional Conference Series in Applied Mathematics.

[24] S. Levin And D. Pimentel, Selection of intermediate rates increase in parasite-host systems, Amer.Naturalist, pp. 308-315 (1981).

[25] S. A. LEvin, Community equilibria and stability, and an extension of the competitive exclusion principle., Amer. Naturalist, 104, pp. 413-423 (1970).

[26] M. Lipsitch AND M. A. NowAK, The evolution of virulence in sexually transmitted hiv/aids, J. Theoret. Biol., 174, pp. 427-440 (1995).

[27] M. Lipsitch, M. A. Nowak, D. Ebert, And R. M. May, The population dynamics of vertically and horizontally transmitted parasites., Proc Biol Sci, 260, pp. 321-327 (1995).

[28] R. MAy, Stability and Complexity in Model Ecosystems, Princeton University Press, 1973.

[29] R. M. MAY AND R. M. Anderson, Epidemiology and genetics in the coevolution of parasites and hosts., Proc R Soc Lond B Biol Sci, 219, pp. 281-313 (1983).

[30] —, Parasite-host coevolution., Parasitology, 100 Suppl, pp. S89-101 (1990).

[31] J. Maynard Smith, Models in Ecology, Cambridge University Press, 1974.

[32] A. R. McLean And R. M. Anderson, Measles in developing countries Part I. Epidemiological parameters and patterns, Epidemiology and Infection, 100, pp. 111-133 (1988).

[33] S. D. Mylius and O. Diekmann, On evolutionarily stable life histories, optimization and the need to be specific about density dependence, Oikos, 74, pp. 218-224 (1995).

[34] H. R. Thieme, Pathogen competition and coexistence and the evolution of virulence, vol. Mathematics for Life Sciences and Medicine, Springer-Verlag, pp. 123-153 (2007).

[35] — Global stability of the endemic equilibrium in infinite dimension : Lyapunov functions and positive operators, J. Differ. Equations, 250, pp. 3772-3801 (2011).

[36] P. VAn Den Driessche And J. WATMOugh, reproduction numbers and sub-threshold endemic equilibria for compartmental models of disease transmission, Math. Biosci., 180, pp. 29-48 (2002).

[37] M. VIDYASAGAR, Decomposition techniques for large-scale systems with nonadditive interactions: Stability and stabilizability., IEEE Trans. Autom. Control, 25, pp. 773-779 (1980).

[38] V. Volterra, Variations and fluctuations of the number of individuals in animal species living together, J. Cons. Cons. Int. Explor. Am. Nat., 3, pp. 3-51 (1928). 